\title{
Competition for Graphene: Graphynes with Direction-Dependent Dirac Cones
}

\author{
Daniel Malko, Christian Neiss, Francesc Viñes, and Andreas Görling \\ Lehrstuhl für Theoretische Chemie and Interdisciplinary Center of Molecular Materials, Universität Erlangen-Nürnberg, \\ Egerlandstr. 3, D-91058 Erlangen, Germany
}

(Received 26 October 2011; published 24 February 2012)

\begin{abstract}
The existence of Dirac cones in the band structure of two-dimensional materials accompanied by unprecedented electronic properties is considered to be a unique feature of graphene related to its hexagonal symmetry. Here, we present other two-dimensional carbon materials, graphynes, that also possess Dirac cones according to first-principles electronic structure calculations. One of these materials, 6,6,12-graphyne, does not have hexagonal symmetry and features two self-doped nonequivalent distorted Dirac cones suggesting electronic properties even more amazing than that of graphene.
\end{abstract}

DOI: 10.1103/PhysRevLett.108.086804

PACS numbers: 73.22. $-\mathrm{f}, 71.15 . \mathrm{Mb}, 73.61 . \mathrm{Ph}$

Since its first preparation [1] graphene, Fig. 1(a), has attracted tremendous interest predominantly due to its extraordinary electronic properties, making it highly interesting from a technical point of view as a promising material for carbon-based electronics [2-12]. Most important is probably the extremely high conductivity of graphene [3-10]. Graphene exhibits ballistic charge transport and enormously high carrier mobilities with comparable conductivities of electrons and holes. The cause of the amazing electronic properties of graphene is its peculiar band structure featuring so-called Dirac points and cones [2-4]. At a Dirac point the valence and conduction bands of graphene meet in a single point at the Fermi level. This means that graphene can be considered as a semiconductor with zero band gap, or, alternatively, as a metal with zero density of states (DOS) at the Fermi level. In the vicinity of a Dirac point the valence and conduction bands form a double cone, the Dirac cone, and approach the Dirac point with zero curvature along certain symmetry-determined directions. Because of its honeycomb structure consisting of two equivalent hexagonal carbon sublattices the electrons in graphene can formally be described by a Dirac-like Hamiltonian operator containing a twodimensional pseudospin operator [2-4]. The applicability of this specific Dirac-like Hamiltonian operator is a peculiarity of the hexagonal honeycomb structure of graphene. However, we show here that the existence of Dirac-conelike features in the band structure of two-dimensional (2D) materials does neither depend on a honeycomb structure nor on hexagonal symmetry. We will adopt the nomenclature to generally denote a double conelike feature where the valence and conduction band of a $2 \mathrm{D}$ material meet in a single point at or very close to the Fermi level Dirac cone provided that valence and conduction band approach the meeting point, then designated as Dirac point, with zero curvature from at least one direction.

So far, the only $2 \mathrm{D}$ carbon allotrope that can be produced or synthesized is graphene [1,10-18]. However, infinitely many other 2D periodic carbon allotropes, e.g., graphynes or graphdiynes, can be envisioned [19-24]. Graphynes and graphdiynes are built from triple- and double-bonded units of two carbon atoms. Examples are displayed in Fig. 1. Many graphynes and graphdiynes including those depicted in Fig. 1 can be assumed to be chemically stable. Indeed, finite building blocks and cutouts have already been synthesized [22,23,25-30], and first steps towards the preparation of extended graphynes and graphdiynes have been proposed and developed $[22,24,26,27]$. Future synthesis of graphyne materials might involve the use of surfaces as support and templates in approaches related to the recently reported synthesis of graphene on metal surfaces by chemical vapor deposition of organic precursor molecules [12-18]. Whether attempts

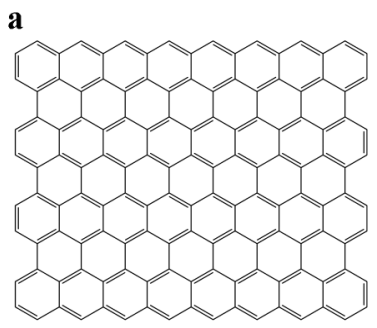

b

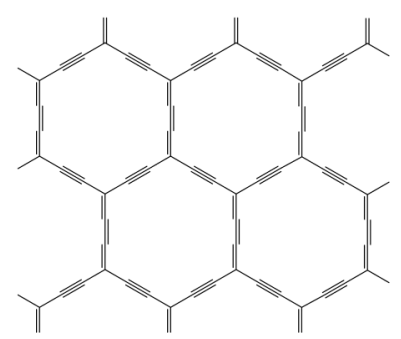

c

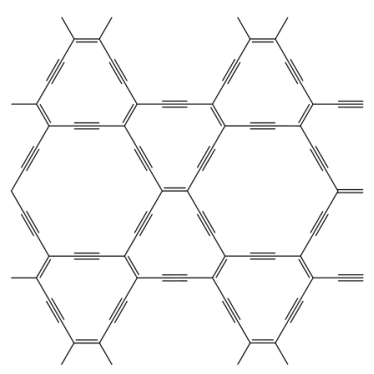

d

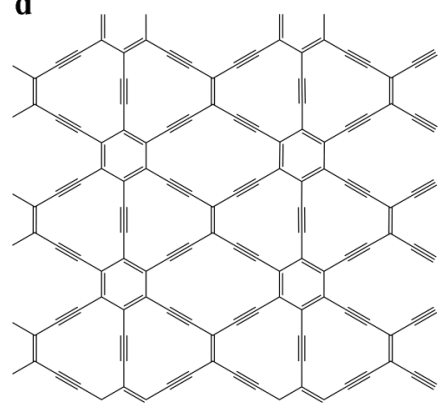

FIG. 1. Structures of graphene and graphynes. (a) Graphene. (b) $\alpha$-graphyne. (c) $\beta$-graphyne. (d) $6,6,12$-graphyne. In all cases only one resonance structure, i.e., one of several equivalent Lewis structures, is shown. 
to synthesize extended graphyne materials are worthwhile depends on the properties such materials would have, in particular, on their electronic properties.

Reliable predictions of material properties require first-principle calculations. In the case of graphynes the methods of choice are based on density functional theory (DFT). Previous DFT studies [20,31-35] concerned with the calculation of electronic properties of graphynes classified them as semiconducting or metallic materials without investigating the possibility of the presence of Dirac cones. In one semiempirical study [36], which employed the density-functional-based tight-binding method, hints on the presence of a graphenelike electronic structure were given for one graphyne. We note in passing that Dirac cones were observed at interfaces of topological insulators [37,38].

In this work we investigate three graphynes, called $\alpha$-graphyne, $\beta$-graphyne, and 6,6,12-graphyne [Figs. 1(b) and 1(c), respectively] by DFT methods in a slab approach using the program package VASP [39] and the exchange-correlation functional according to Perdew, Burke, and Ernzerhof [40]; for details see Supplemental Material [41]. For each of these graphynes, we first carried out a geometry optimization. In every case, we confirmed by a vibrational analysis that the optimized structures are stable, i.e., constitute minima on the potential energy surface. In the next step, band structures and DOSs were calculated.

The band structures and DOSs of $\alpha$-graphyne and $\beta$-graphyne (Fig. 2 and 3) show that $\alpha$-graphyne has two and $\beta$-graphyne has six points in the Brillouin zone where valence and conduction band meet in a single point at the Fermi level. (As the two, respectively, six points are related by symmetry, only one representative is shown in Figs. 2 and 3.) Consistently, the DOS of $\alpha$-graphyne and $\beta$-graphyne is zero at the Fermi level [Figs. 2(b) and 3(b)]. For $\alpha$-graphyne these meeting points of valence and conduction bands are Dirac points equivalent to those of graphene and are located at the same positions in the Brillouin zone, the $K$ and $K^{\prime}$ points. The slopes and curvatures, i.e., first and second derivatives of the band energies of the two involved bands in reciprocal space, equal $\pm 28 \mathrm{eV} \AA$ and $\pm 250 \mathrm{eV} \AA^{2}$ compared to $\pm 34 \mathrm{eV} \AA$ and $\pm 130 \mathrm{eV} \AA^{2}$ in graphene when approaching a Dirac point on a line through the $\Gamma$ and $K$ point, respectively, the $\Gamma$ and $K^{\prime}$ point. When approaching a Dirac point along lines perpendicular to the former ones the slopes essentially remain unchanged while the curvatures are zero. In $\beta$-graphyne, on the other hand, the meeting points of valence and conduction bands are Dirac points not located at the $K$ or $K^{\prime}$ point but on lines from $\Gamma$ to $M$. If the Dirac point is approached along the latter line then the two involved bands exhibit curvatures of +800 and $-840 \mathrm{eV} \AA^{2}$. (The corresponding slopes equal -16 and $+18 \mathrm{eV} \AA$.) Perpendicular to the line from $\Gamma$ to $M$, which
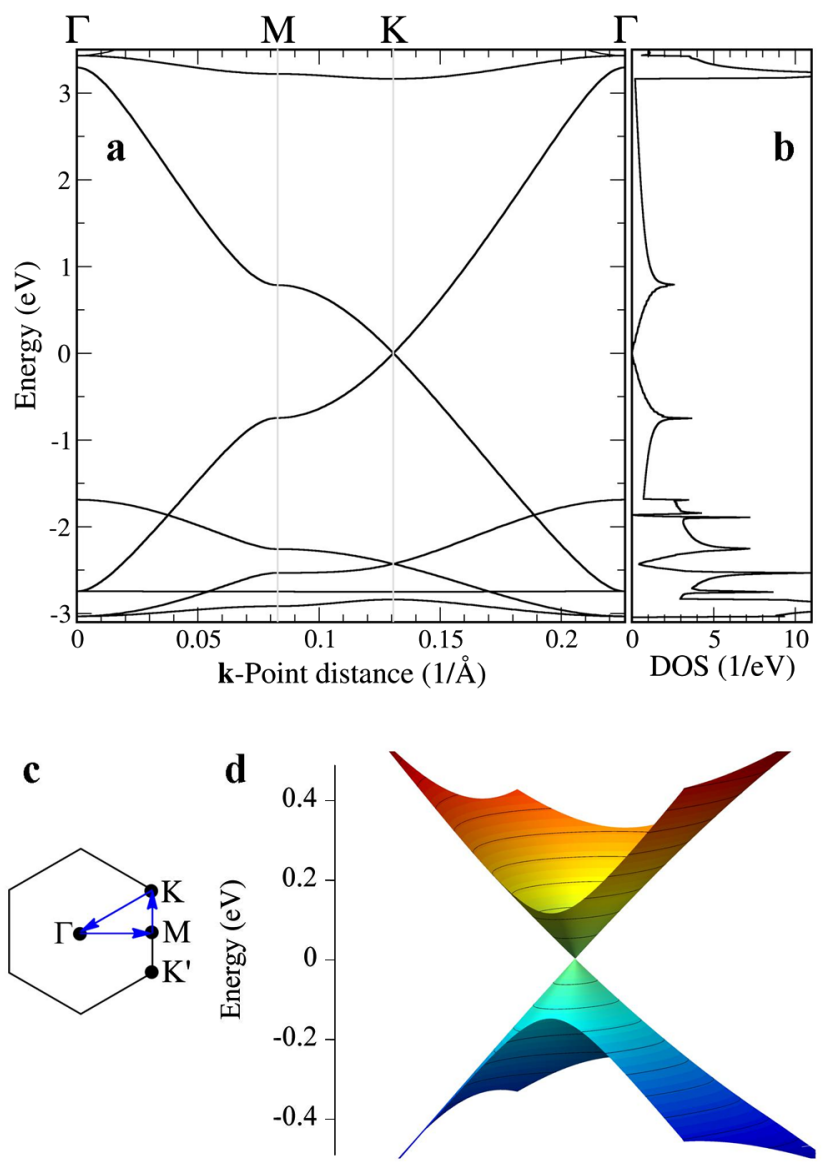

FIG. 2 (color online). Electronic structure of $\alpha$-graphyne. (a) Band structure. (b) DOS. (c) First Brillouin zone with letters designating special points and with the lines along which the band structure is displayed. (d) Dirac cone formed by the valence and conduction band in the vicinity of the Dirac point.

is a mirror line in reciprocal space for $\beta$-graphyne, the valence and conduction bands approach a Dirac point with zero curvature and slopes of $\pm 28 \mathrm{eV} \AA$.

The Dirac cones of $\alpha$-graphyne and $\beta$-graphyne exhibit different symmetries. In $\alpha$-graphyne the Dirac cones, due to their location at the $K$ and $K^{\prime}$ point, have a threefold symmetry like in graphene. This means valence and conduction bands have zero curvature not only along a line perpendicular to the $\Gamma$ - $K$, respectively $\Gamma-K^{\prime}$, direction, but also along lines obtained by rotating these lines by $120^{\circ}$ and $240^{\circ}$. The two Dirac points of $\alpha$-graphyne are rotated by an angle of $60^{\circ}$ against each other. In $\beta$-graphyne, on the other hand, one of the six Dirac cones exhibits reflection symmetry with respect to the $\Gamma-M$ direction and the valence and conduction bands have zero curvature only along the line perpendicular to this direction. The other five Dirac cones of $\beta$-graphyne are symmetry related by rotations of the Brillouin zone in steps of $60^{\circ}$. In both cases the hexagonal symmetry prevents a significant directional dependence of the electronic properties in the plane of the material. Despite their differences in symmetry, 2D plots of 

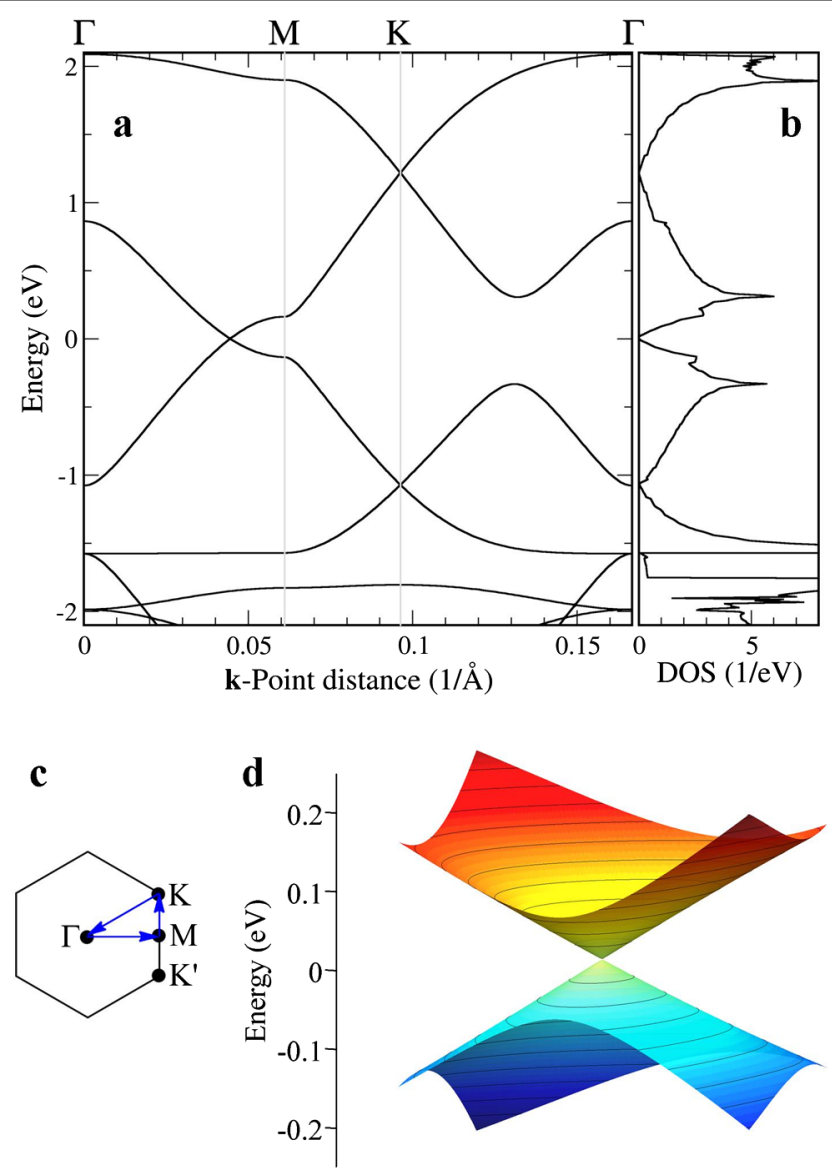

FIG. 3 (color online). Electronic structure of $\beta$-graphyne. (a) Band structure. (b) DOS. (c) First Brillouin zone with letters designating special points and with the lines along which the band structure is displayed. (d) Dirac cone formed by the valence and conduction band in the vicinity of the Dirac point.

the Dirac cones of both $\alpha$-graphyne and $\beta$-graphyne [Figs. 2(d) and 3(d)] exhibit a similar appearance. Charge density plots of the orbitals (one-electron wave-functions) at the Dirac points can be found in the Supplemental Material [41] for graphene and all investigated graphynes.

Both $\alpha$-graphyne and $\beta$-graphyne exhibit hexagonal lattice symmetry $(p 6 m)$ like graphene. This symmetry is commonly considered a necessary precondition for the presence of Dirac cones. The band structure of 6,6,12graphyne (Fig. 4) shows that this is not the case. In contrast to graphene or $\alpha$-graphyne and $\beta$-graphyne, $6,6,12$ graphyne has a rectangular $(\mathrm{pmm})$ instead of a hexagonal symmetry. Nevertheless, 6,6,12-graphyne exhibits four Dirac points in its Brillouin zone which appear in two pairs, hereafter denoted as Dirac points I and II. The Dirac points of a given pair are symmetry related, those of different pairs are not. Only one of the two Dirac points of a given pair is shown in Fig. 4. The orbitals (one-electron wave functions) at the two types of Dirac points are located at different carbon atoms as can be seen from their charge density plots; see the Supplemental Material [41].
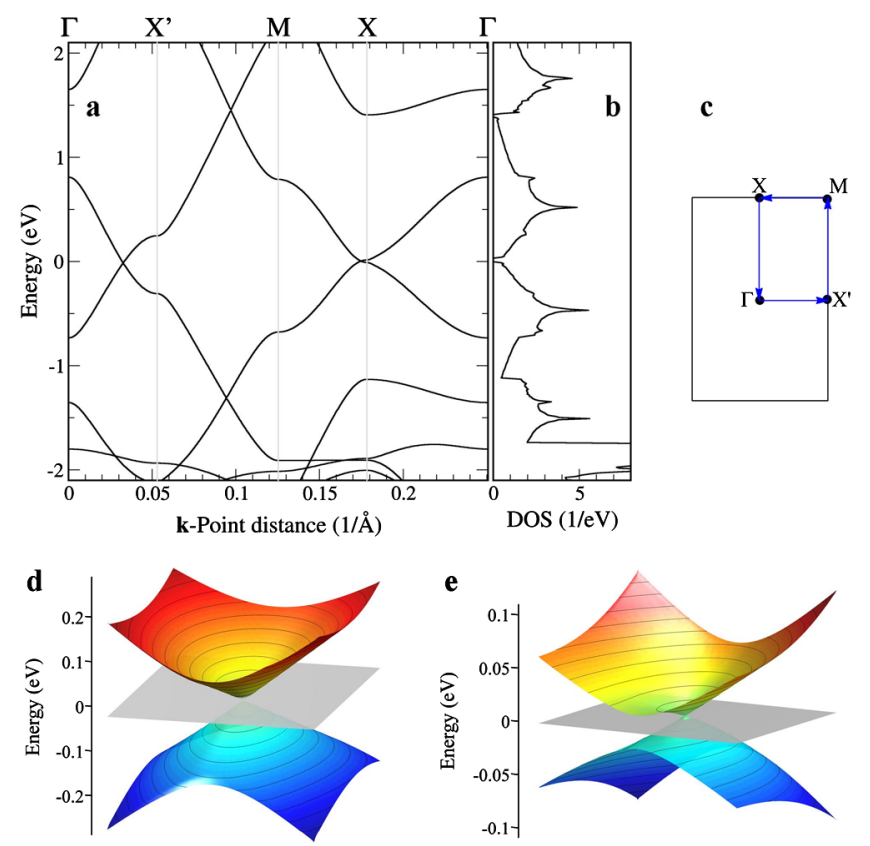

FIG. 4 (color online). Electronic structure of 6,6,12-graphyne. (a) Band structure. The two different Dirac points are labeled by I and II. (b) DOS. (c) First Brillouin zone with letters designating special points and with the lines along which the band structure is displayed. (d) Dirac cone I. (e) Dirac cone II. Gray planes in panels (d) and (e) indicate the Fermi level.

The valence and the conduction bands in the vicinity of the two types of Dirac points [Figs. 4(d) and 4(e)] show the presence of distorted Dirac cones. (A two-dimensional plot of the band structure for the complete irreducible part of the Brillouin zone can be found in the Supplemental Material [41].) Around both Dirac points the valence and conduction bands exhibit a linear dispersion in the $k_{y}$ direction, the direction from $\Gamma$ to $X$, whereas the bands show a finite curvature in the $k_{x}$ direction, the direction from $\Gamma$ to $X^{\prime}$. The slope of the bands in the $k_{y}$ direction equals $\pm 25 \mathrm{eV} \AA$ at Dirac cone I and $\pm 9 \mathrm{eV} \AA$ at Dirac cone II while the curvatures are zero. In the $k_{x}$ direction the slopes of the bands are $+23 \mathrm{eV} \AA$ and $-26 \mathrm{eV} \AA$ at Dirac point I and $\pm 7 \mathrm{eV} \AA$ at Dirac point II while the curvatures equal $\pm 690 \mathrm{eV} \AA^{2}$ at Dirac point I and $\pm 2000 \mathrm{eV} \AA^{2}$ at Dirac point II. The shape of the Dirac cones is related to the rectangular symmetry of 6,6,12-graphyne. The lines from $\Gamma$ to $X^{\prime}$ and from $X$ to $M$, where the distorted Dirac points are located at, are mirror lines in reciprocal space for 6,6,12-graphyne. The two lines run in the $k_{x}$ direction and determine the $k_{y}$ position of the Dirac cones while the $k_{x}$ position of the Dirac cones is not determined by symmetry. The rectangular symmetry, in particular, the distortion of the Dirac cones with different slopes and curvatures at the Dirac points in the $k_{x}$ and $k_{y}$ directions going along with it, implies direction-dependent electronic properties, notably conductivities. 
Interestingly, Dirac cone I lies slightly below the Fermi level while Dirac cone II lies slightly above the Fermi level [Figs. 4(d) and 4(e)]. This means that 6,6,12-graphyne is self-doped in the sense that at Dirac cone I electrons are present as charge carriers while at Dirac cone II holes are present as charge carriers. Because the two Dirac points of 6,6,12-graphyne are different with respect to shape and doping they will contribute differently to the electronic properties.

The example of $\alpha$-graphyne shows that the existence of Dirac points and cones is not a unique feature of graphene. Indeed, $\alpha$-graphyne is a material that in contrast to graphene contains carbon atoms in different chemical environments. Thus, the fact that all carbon atoms are chemically equivalent in graphene is not a prerequisite for the existence of a Dirac cone and accompanying electronic properties, like outstanding conductivity. The results for $\beta$-graphyne demonstrate that Dirac cones not only can occur at the high-symmetry $K$ and $K^{\prime}$ points but also at low-symmetry points in the Brillouin zone of 2D materials with hexagonal lattice symmetry. The example of 6,6,12graphyne finally shows that even hexagonal symmetry is not a prerequisite for Dirac points. In the case of 6,6,12graphyne a linear dispersion of the bands in the $k_{y}$ direction at its distorted Dirac points is related to its rectangular symmetry. This indicates that many 2D materials with various symmetries, probably even $2 \mathrm{D}$ materials that are made out of other atoms than carbon, could have Dirac points or distorted Dirac points and, as a result, could possess highly interesting electronic properties. In general, the examples presented here suggest that a wealth of $2 \mathrm{D}$ materials with electronic properties that might be similarly amazing and promising as those of graphene is waiting to be synthesized and applied. The specific example of 6,6,12-graphyne indicates that this material in some aspects is more versatile than graphene, due to its directional anisotropy and its nonequivalent Dirac points.

This work was funded by the German Research Council (DFG) via the Collaborative Research Center 953 and via the Cluster of Excellence "Engineering of Advanced Materials"[42] at the University of Erlangen-Nuremberg. F. V. thanks the Alexander von Humboldt foundation for his postdoctoral grant.

[1] K. S. Novoselov et al., Science 306, 666 (2004).

[2] A. K. Geim, Science 324, 1530 (2009).

[3] A. K. Geim and K.S. Novoselov, Nature Mater. 6, 183 (2007).

[4] K. S. Novoselov et al., Nature (London) 438, 197 (2005).

[5] Y. Zhang, Y.-W. Tan, H. L. Stormer, and P. Kim, Nature (London) 438, 201 (2005).

[6] N. M. R. Peres, F. Guinea, and A. H. Castro Neto, Phys. Rev. B 73, 125411 (2006).

[7] C. Berger et al., Science 312, 1191 (2006).

[8] M. Orlita et al., Phys. Rev. Lett. 101, 267601 (2008).
[9] A. Bostwick, T. Otha, T. Seyller, K. Hom, and E. Rotenberg, Nature Phys. 3, 36 (2006).

[10] K. V. Emtsev et al., Nature Mater. 8, 203 (2009).

[11] P. W. Sutter, J.-I. Flege, and E. A. Sutter, Nature Mater. 7, 406 (2008).

[12] K. S. Kim et al., Nature (London) 457, 706 (2009).

[13] T. A. Land, T. Michely, R. J. Behm, J. C. Hemminger, and G. Comsa, Surf. Sci. 264, 261 (1992).

[14] C. Oshima and A. Nagashima, J. Phys. Condens. Matter 9 , 1 (1997).

[15] A. Varykhalov et al., Phys. Rev. Lett. 101, 157601 (2008).

[16] D. Martoccia et al., Phys. Rev. Lett. 101, 126102 (2008).

[17] A. Grüneis, K. Kummer, and D. V. Vyalikh, New J. Phys. 11, 073050 (2009).

[18] J. Wintterlin and M.L. Bocquet, Surf. Sci. 603, 1841 (2009).

[19] R. H. Baughman and H. Eckhardt, J. Chem. Phys. 87, 6687 (1987).

[20] V. R. Coluci, S. F. Braga, S. B. Legoas, D. S. Galvão, and R. H. Baughman, Phys. Rev. B 68, 035430 (2003).

[21] A. Hirsch, Nature Mater. 9, 868 (2010).

[22] F. Diederich, Nature (London) 369, 199 (1994).

[23] U. H. F. Bunz, Y. Rubin, and Y. Tobe, Chem. Soc. Rev. 28, 107 (1999).

[24] G. Li et al., Chem. Commun. (Cambridge) 46, 3256 (2010).

[25] J. M. Kehoe et al., Org. Lett. 2, 969 (2000).

[26] J. A. Marsden, G. J. Palmer, and M. M. Haley, Eur. J. Org. Chem. 2003, 2355 (2003).

[27] M. Gholami et al., Angew. Chem., Int. Ed. 46, 9081 (2007).

[28] T. Yoshimura et al., Org. Lett. 8, 2933 (2006).

[29] M. M. Haley, Pure Appl. Chem. 80, 519 (2008).

[30] F. Diederich and M. Kivala, Adv. Mater. 22, 803 (2010).

[31] N. Narita, S. Nagai, S. Suzuki, and K. Nakao, Phys. Rev. B 58, 11009 (1998).

[32] J. Zhou et al., J. Chem. Phys. 134, 174701 (2011).

[33] E. Konstantinova, S. O. Dantas, and P. M. V. B. Barone, Phys. Rev. B 74, 035417 (2006).

[34] M. Long, L. Tang, D. Wang, Y. Li, and Z. Shuai, ACS Nano 5, 2593 (2011).

[35] L. D. Pan, L.Z. Zhang, B. Q. Song, S.X. Du, and H.-J. Gao, Appl. Phys. Lett. 98, 173102 (2011).

[36] A. N. Enyashin and A. L. Ivanovskii, Phys. Status Solidi B 248, 1879 (2011).

[37] L. Fu, C. L. Kane, and E. J. Mele, Phys. Rev. Lett. 98, 106803 (2007).

[38] R. Takahashi and S. Murakami, Phys. Rev. Lett. 107, 166805 (2011).

[39] G. Kresse and J. Furthmüller, Phys. Rev. B 54, 11169 (1996).

[40] J. P. Perdew, K. Burke, and M. Ernzerhof, Phys. Rev. Lett. 77, 3865 (1996).

[41] See Supplemental Material at http://link.aps.org/ supplemental/10.1103/PhysRevLett.108.086804 for computational details, orbital density plots of graphene and graphynes, and a two-dimensional plot of the valence and conduction band of 6,6,12-graphyne within the complete irreducible part of the Brillouin zone.

[42] www.eam.uni-erlangen.de 